Bull. Korean Math. Soc. 45 (2008), No. 4, pp. 781-795

\title{
ADAPTIVE MESH REFINEMENT FOR WEIGHTED ESSENTIALLY NON-OSCILLATORY SCHEMES
}

\author{
Daeki Yoon, Hongjoong Kim, and Woonjae Hwang
}

\begin{abstract}
In this paper, we describe the application procedure of the adaptive mesh refinement (AMR) for the weighted essentially non-oscillatory schemes (WENO), and observe the effects of the derived algorithm when problems have piecewise smooth solutions containing discontinuities. We find numerically that the dissipation of the WENO scheme can be lessened by the implementation of AMR while the accuracy is maintained. We deduce from the experiments that the AMR-implemented WENO scheme captures shocks more efficiently than the WENO method using uniform grids.
\end{abstract}

\section{Introduction}

In this study, we describe numerical schemes for one-dimensional conservation laws

$$
\frac{\partial u}{\partial t}+\frac{\partial f(u)}{\partial x}=0,(x, t) \in I \times[0, \infty),
$$

where $I$ is an interval in space. The weighted essentially non-oscillatory (WENO) scheme is a high order accurate finite difference scheme for problems with piecewise smooth solutions containing discontinuities $[1,2,3]$. There are two approaches of the WENO scheme to solve (1). One is the finite difference WENO, which solves discrete version of (1), while the finite volume approach does not solve (1) directly but its integrated version. That is, the finite difference approach evolves point values whereas the finite volume approach evolves cell averages. Balsara and Shu [4] derived finite difference WENO schemes with orders from 7 to 13 for one dimensional problems. For high dimensional problems, the computational cost for the finite volume WENO approach is more expensive than that of the finite difference WENO approach $[3,5]$. In addition, the

Received April 1, 2008; Revised July 24, 2008.

2000 Mathematics Subject Classification. Primary 65M06, 65M50, 35L65.

Key words and phrases. WENO scheme, adaptive mesh refinement, conservation laws.

This work of H. Kim was supported by the Korea Research Foundation Grant funded by the Korean Government (MOEHRD) (KRF-2007-331-C00053).

This work of W. Hwang was supported by a Korea University Grant. 
finite volume approach may cause negative weights for high dimensional problems [5]. See $[3,6]$ for detailed comparison between these approaches. WENO scheme has been an effective method to problems containing shocks or complicated solution structures. Even though WENO can resolve those fine-scale features for unsteady flows, numerical solutions are slightly dissipative. Several approaches have been introduced by many researchers in order to reduce those dissipative errors. See [2] and references therein.

This paper is mainly concerned with reducing numerical errors of the WENO scheme by implementing the adaptive mesh refinement (AMR) to the scheme. AMR is a numerical method by which the computational domain is discretized into grids of different sizes. This numerical scheme gives high resolution to solutions by automatically refining regions where high resolution is required $[7,8]$. Two types of refinements are available, static or dynamic. Static mesh is set up in the beginning of the computation based on static characteristics of the domain such as the permeability field of the porous medium [9], and is used throughout the computation without any modification. On the other hand, many different meshes in time are allowed in case of dynamic refinements. Adaptive refinement of grids plays an important role in reducing computational costs. This study implements the advantages of AMR for WENO so that complicated solutions can be identified while unnecessary calculations are avoided. Recently Wang et al in [6] have derived explicit formulas for the finite-volume, fifth-order WENO method on non-uniform meshes. Their research shows improvements on the computational efficiency over uniform mesh approach. However, since the fine mesh is chosen a priori, the scheme introduced in [6] can be applicable only for short period of time. In addition, it seems to be difficult to apply the scheme to high dimensional problems.

This paper is organized as follows: In Section 2, we review basic facts of the WENO and AMR methods. Section 3 performs several numerical experiments, in which AMR-implemented WENO scheme is compared with the WENO, especially in view of the efficiency. In fact, it has been shown that the computational cost is saved by introducing AMR. Concluding remarks and future research directions are pointed out in Conclusions.

\section{Preliminaries on WENO and AMR}

\subsection{WENO}

In this section, we explore the WENO scheme [3]. We first consider a onedimensional reconstruction problem using the WENO scheme and then apply the scheme to one-dimensional conservation laws.

2.1.1. One-dimensional reconstruction problem using WENO. Given a closed interval $[a, b]$, we divide the interval into $N$ uniform subintervals

$$
a=x_{\frac{1}{2}}<x_{\frac{3}{2}}<\cdots<x_{N-\frac{1}{2}}<x_{N+\frac{1}{2}}=b
$$


where $x_{i+\frac{1}{2}}=x_{\frac{1}{2}}+i \Delta x$ for the index $i=1,2, \ldots, N$ and $\Delta x=\frac{b-a}{N}$. The point $x_{i}=\frac{1}{2}\left(x_{i-\frac{1}{2}}+x_{i+\frac{1}{2}}\right)$ is called the center of the $i^{\text {th }}$ cell $I_{i}=\left[x_{i-\frac{1}{2}}, x_{i+\frac{1}{2}}\right]$. Given the cell averages $\left\{\bar{v}_{i}\right\}$ of a function $v(x)$,

$$
\bar{v}_{i}=\frac{1}{\Delta x} \int_{x_{i-\frac{1}{2}}}^{x_{i+\frac{1}{2}}} v(\xi) d \xi
$$

for each cell $I_{i}$, the $k^{\text {th }}$ order approximations to $v(x)$ at the cell boundaries, denoted by $v_{i-\frac{1}{2}}^{+}$and $v_{i+\frac{1}{2}}^{-}$are calculated in the following way. Let $k=5$ in our study below. First, $v_{i+\frac{1}{2}}^{(r)}$ and $v_{i-\frac{1}{2}}^{(r)}$ are obtained by

$$
v_{i+\frac{1}{2}}^{(r)}=\sum_{j=0}^{k-1} c_{r, j} \bar{v}_{i-r+j},
$$

and

$$
v_{i-\frac{1}{2}}^{(r)}=\sum_{j=0}^{k-1} c_{r-1, j} \bar{v}_{i-r+j}
$$

for $r=0,1,2$. The coefficient $c_{r, j}$ is obtained from the following Table 1 :

TABLE 1. The coefficient $c_{r, j}$ in (2) and (3)

\begin{tabular}{c|ccc}
$r$ & $j=0$ & $j=1$ & $j=2$ \\
\hline-1 & $11 / 6$ & $-7 / 6$ & $1 / 3$ \\
0 & $1 / 3$ & $5 / 6$ & $-1 / 6$ \\
1 & $-1 / 6$ & $5 / 6$ & $1 / 3$ \\
2 & $1 / 3$ & $-7 / 6$ & $11 / 6$
\end{tabular}

Let us define $\beta_{0}, \beta_{1}$ and $\beta_{2}$ by

$$
\begin{aligned}
& \beta_{0}=\frac{13}{12}\left(\bar{v}_{i}-2 \bar{v}_{i+1}+\bar{v}_{i+2}\right)^{2}+\frac{1}{4}\left(3 \bar{v}_{i}-4 \bar{v}_{i+1}+\bar{v}_{i+2}\right)^{2} \\
& \beta_{1}=\frac{13}{12}\left(\bar{v}_{i-1}-2 \bar{v}_{i}+\bar{v}_{i+1}\right)^{2}+\frac{1}{4}\left(\bar{v}_{i-1}-\bar{v}_{i+1}\right)^{2} \\
& \beta_{2}=\frac{13}{12}\left(\bar{v}_{i-2}-2 \bar{v}_{i-1}+\bar{v}_{i}\right)^{2}+\frac{1}{4}\left(\bar{v}_{i-2}-4 \bar{v}_{i-1}+3 \bar{v}_{i}\right)^{2} .
\end{aligned}
$$

Let $d_{0}=\frac{3}{10}, d_{1}=\frac{3}{5}$, and $d_{2}=\frac{1}{10}$. Then $w_{r}$ and $\tilde{w}_{r}$ are defined by

$$
w_{r}=\frac{\alpha_{r}}{\sum_{s=0}^{2} \alpha_{s}} \text { and } \tilde{w}_{r}=\frac{\tilde{\alpha}_{r}}{\sum_{s=0}^{2} \tilde{\alpha}_{s}}
$$

respectively, where $\alpha_{r}=\frac{d_{r}}{\left(\epsilon+\beta_{r}\right)^{2}}$, and $\tilde{\alpha}_{r}=\frac{d_{2-r}}{\left(\epsilon+\beta_{r}\right)^{2}} \cdot \epsilon=10^{-6}$ is used in the denominators to avoid the division by zero [3]. Finally $v_{i-\frac{1}{2}}^{+}$and $v_{i+\frac{1}{2}}^{-}$can be 
reconstructed by

$$
v_{i+\frac{1}{2}}^{-}=\sum_{r=0}^{2} w_{r} v_{i+\frac{1}{2}}^{(r)} \text { and } v_{i-\frac{1}{2}}^{+}=\sum_{r=0}^{2} \tilde{w}_{r} v_{i-\frac{1}{2}}^{(r)} .
$$

2.1.2. Time discretization of WENO scheme. An ordinary differential equation

$$
\frac{d u}{d t}=L(u)
$$

where $L(u)$ is a discretization of the spatial differential operator, can be solved by the third-order TVD Runge-Kutta method in [1] to preserve the conservation law:

$$
\begin{aligned}
u^{(1)} & =u^{n}+\Delta t L\left(u^{n}\right), \\
u^{(2)} & =\frac{3}{4} u^{n}+\frac{1}{4} u^{(1)}+\frac{1}{4} \Delta t L\left(u^{(1)}\right), \\
u^{n+1} & =\frac{1}{3} u^{n}+\frac{2}{3} u^{(2)}+\frac{2}{3} \Delta t L\left(u^{(2)}\right) .
\end{aligned}
$$

The one-dimensional scalar conservation laws (1) can be converted to an ordinary differential equation (5) in time above if the spatial derivative $L$ is discretized by

$$
L=-\frac{1}{\Delta x}\left(\hat{f}_{i+\frac{1}{2}}-\hat{f}_{i-\frac{1}{2}}\right),
$$

where the numerical flux $\hat{f}_{i+\frac{1}{2}}$ approximates $h_{i+\frac{1}{2}}=h\left(x_{i+\frac{1}{2}}\right)$ to a high order with $h(x)$ implicitly defined by

$$
f(u(x))=\frac{1}{\Delta x} \int_{x-\Delta x / 2}^{x+\Delta x / 2} h(\xi) d \xi
$$

as in $[1,10]$.

\subsection{AMR}

AMR introduces more than one scales to represent variables in space or time. When the AMR flags a variable in some region, the variable is redefined on finer scales there. That is, given a grid and a solution on it, when the solution requires higher resolution, we first flag those cells that require refinement, and then evaluate the solution on the new finer grids. R. Deiterding [11] explains AMR mathematically, especially on its parallel implementation. It also explains how to handle multi-dimensional problems using AMR. M. Berger and I. Rigoutsos [12] explains methods for flagging and generating new rectangular grids. See also [13].

At each time if a solution on some level requires higher resolution, flagging has been introduced over the corresponding grid. In this study, two measures have been used for flagging. First, the gradient $|\nabla u|$ is estimated to measure discontinuity. If this gradient is large, the solution may contain a discontinuity 
and the corresponding grid has been flagged. Secondly, even when the gradient is small if solutions from two different step sizes have large deviations, then the corresponding grid has been also flagged. That is, if $\left\|u_{\Delta x}(x, t)-u_{2 \Delta x}(x, t)\right\|_{\infty}$ is large, the grid has been refined, where $u_{k}$ is the solution from the difference scheme with step size $k$ and $\|\cdot\|_{\infty}$ is the $L^{\infty}$ error. Grids on this flagged region is replaced by new finer grids. When new grids are created, if the values of the solution on those grids can be calculated from the solution of previous time step, those calculated values are used on the fine grids. On the other hand, if the solution on those fine grids are not defined previously, interpolations are used to assign values on the corresponding cells. If the periodic boundary condition is given, interpolations are performed so that periodicity is maintained at the boundaries [14]. Linear interpolations are used in this study because oscillations occur near discontinuities when the second or higher order interpolations are used. Since the AMR performs extra computation over a flagged region only, it may cause significant decrease of the computational costs such as CPU time over non-AMR schemes.

Grids for each resolution are called levels. Level 0 implies the coarsest grids covering the computational domain. Level 1 consists of fine grids for a solution on grids of level 0, which requires higher resolution. Level 2 consists of fine grids for a solution on grids of level 1, which requires higher resolution. Higher levels are defined in a similar way. Thus, more accurate solutions can be obtained on finer grids. In our study, rectangular grids are used on each level.

Since AMR uses several different grids in space, solutions on spatial grids of each level have different timesteps when the CFL number is fixed. Let $\Delta x_{i}$ and $\Delta t_{i}$ be the cell sizes in space and time of the $i^{\text {th }}$ level, respectively, such that $\Delta t_{i} / \Delta x_{i}$ is fixed and $\Delta x_{i}=\Delta x_{i-1} / 2$ for $i=1,2, \ldots$. For simplicity, let us assume that we have 4 levels, but the analysis below can be applied to any number of levels. Suppose that the solutions on each level are at time $t$. First the solution on level 0 propagates by $\Delta t_{0}$ in time. Secondly the solution on level 1 propagates by $\Delta t_{1}$ once. Then the solution on level 2 propagates once. Finally the solution on level 3 propagates once. After the propagation on level 3 is performed, the propagation on this finest grid is performed once more to reach $t+2 \Delta t_{3}=t+\Delta t_{2}$. Then the solution on level 2 propagates once more to reach $t+2 \Delta t_{2}=t+\Delta t_{1}$, and the solution on level 3 propagates twice to reach $t+\Delta t_{1}$. After that the solution on level 1 moves by $\Delta t_{1}$ and solutions on higher levels move the same way as above. When solutions on all the levels reach $t+\Delta t_{0}$, they propagate to $t+2 \Delta t_{0}$ in a similar way. This process is repeated until the final time is reached $[11,15]$. See Figure 1.

In this study, when a new level in hierarchy is introduced, grids in the previous level are divided into 4 smaller grids due to the ghost cells at the boundaries from the WENO scheme. When levels are introduced at each time step, they are increased up to 2 higher levels, even though levels can be increased to any number of levels. 


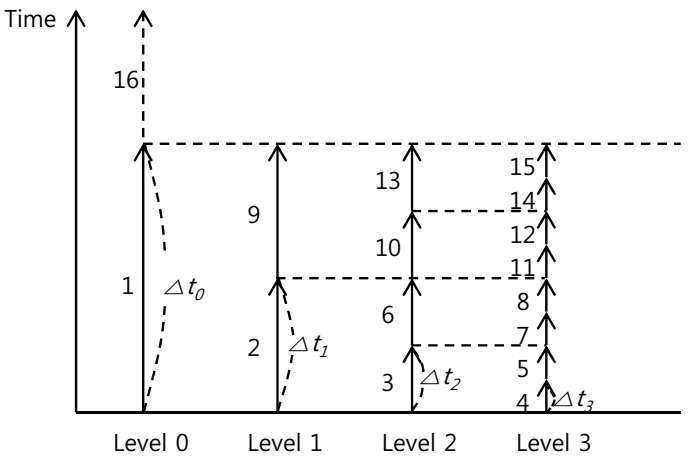

Figure 1. Time evolution of AMR.

\section{Numerical examples}

In this section, we perform several experiments to evaluate the AMR-implemented WENO method. First, the simple linear advection equation is considered. Then the derived scheme is applied to the nonlinear Burgers' equation and a nonlinear system of Euler equations.

\subsection{D linear advection}

Let us consider the Riemann problem for the one-dimensional linear advection equation:

$$
\begin{aligned}
& u_{t}+u_{x}=0, x \in[-1,1], \\
& u(x, 0)= \begin{cases}2 & \text { if } x<0 \\
1 & \text { if } x \geq 0\end{cases}
\end{aligned}
$$

The WENO scheme with various values of $N$ has been applied to solve the problem for $t \leq 0.5$. The CFL number is fixed to 0.5. As pointed out in [2], WENO captures shocks or contact discontinuities accurately, but it introduces numerical dissipation. Figure 2 represents the exact solution and the numerical solution from the WENO scheme with $N=50$ uniform grid points. It shows that the WENO scheme introduces numerical dissipation near $x=0.5$ where a discontinuity develops. As the grid size is reduced, numerical diffusion from the WENO is decreased. For example, the solution from the WENO scheme with $N=800$ points in Figure 4 shows much less diffusion than that observed in Figure 2.

In order to estimate the efficiency of the derived AMR-implemented WENO scheme, we compare the numerical result of the WENO scheme with that of the AMR-implemented WENO, whose smallest grid is the same as the uniform grid of the WENO. Since each level of mesh refinement introduces 4 finer grids in this study and the current AMR experiments allow up to 2 higher levels, numerical 


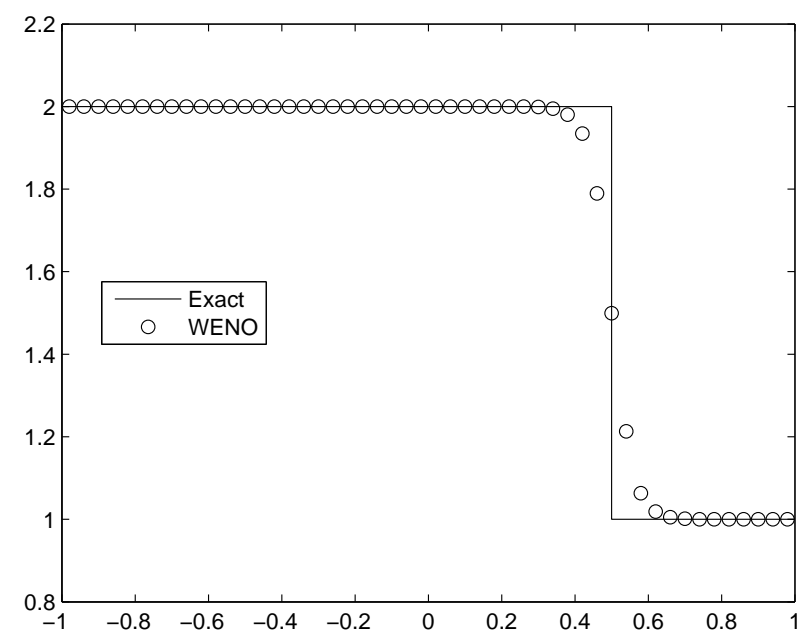

FiguRE 2. Exact solution to the $1 \mathrm{D}$ advection problem at $t=0.5$ and the numerical solution from the WENO scheme with $N=50$.

results from the AMR-implemented WENO with $N$ points are compared with those from the WENO with $4^{2} N$ points. Figure 3 shows a portion of grids in time, introduced by the refinement algorithm. Finer grids are shown near a discontinuity as expected.

Figure 4 shows the solutions to the linear advection problem at $t=0.5$ from the WENO scheme with $N=800$ uniform grid points and the AMRimplemented WENO scheme with $N=50$ points. The AMR-implemented WENO scheme introduces extra grids near a discontinuity, which result in accurate shock capturing. The figure shows that the accuracy from the AMRimplemented WENO with $N=50$ is akin to that from the WENO with $N=$ 800. Numerical diffusion from the WENO in Figure 2 is reduced when fine grids are applied and the introduction of AMR has the same effects.

Table 2 shows the computational costs for the WENO and AMR-implemented WENO schemes. The cost is measured by the CPU time required for the computation. The programs are written in Java and the computations are performed on a computer with Intel Core $22.0 \mathrm{GHz}$ CPU. Table 2 and Figure 4 show that the accuracy is comparable to that of the WENO with $N=800$ uniform grids, while about $90 \%$ of unnecessary computations have been saved when AMR is implemented in case of the one-dimensional linear advection problem. 


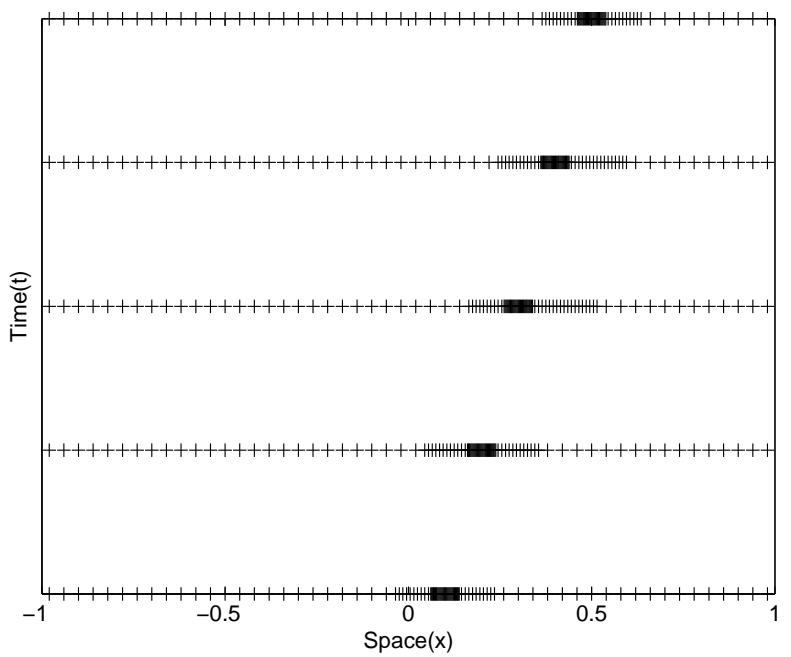

Figure 3. Grids in time used for the 1D advection problem.

TABLE 2. Computational costs for the WENO and AMRimplemented WENO schemes for the 1D linear advection problem for $t \leq 0.5$

\begin{tabular}{c|cc}
$t$ & WENO $(N=800)$ & $\operatorname{AMR}(N=50)$ \\
\hline 0.5 & $2 \sec 187 \mathrm{~ms}$ & $219 \mathrm{~ms}$ \\
\hline
\end{tabular}

\subsection{Burgers' equation}

We test the efficiency of the AMR-implemented WENO scheme in this section on the nonlinear Burgers' equation

$$
\begin{aligned}
u_{t}+\left(\frac{1}{2} u^{2}\right)_{x} & =0, x \in[-1,1] \\
u(x, 0) & =0.3+0.1 \sin (\pi x)
\end{aligned}
$$

with periodic boundary conditions. The CFL number is fixed to 0.5.

Solutions of the above Burgers' equation at early times are smooth and Figure 5 shows solutions at $t=1$. The figure shows that the AMR-implemented WENO with $N=50$ points give the sufficient resolution for the smooth solution.

Shocks will develop in time and Figure 6 shows solutions containing shocks at $t=6$ from the WENO scheme with $N=800$ uniform grid points and the AMR-implemented WENO with $N=50$ points. With only two levels of 

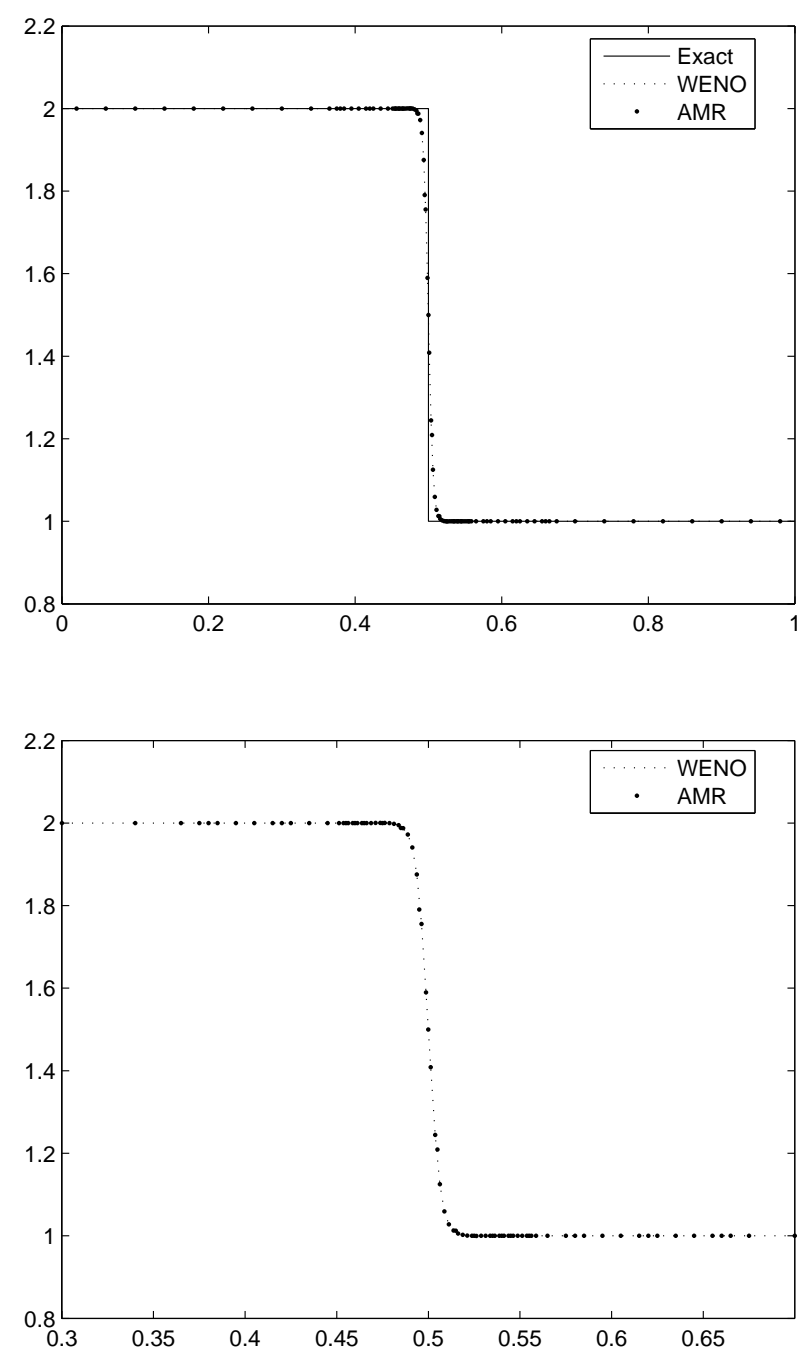

Figure 4. (Top) Exact solution to the 1D advection problem at $t=0.5$ and numerical solutions from the WENO scheme with $N=800$ points and from the AMR-implemented WENO scheme with $N=50$ points and (Bottom) the magnification near a shock about $x=0.5$.

refinements, AMR can capture shocks as accurately as WENO with much finer uniform grids does. 


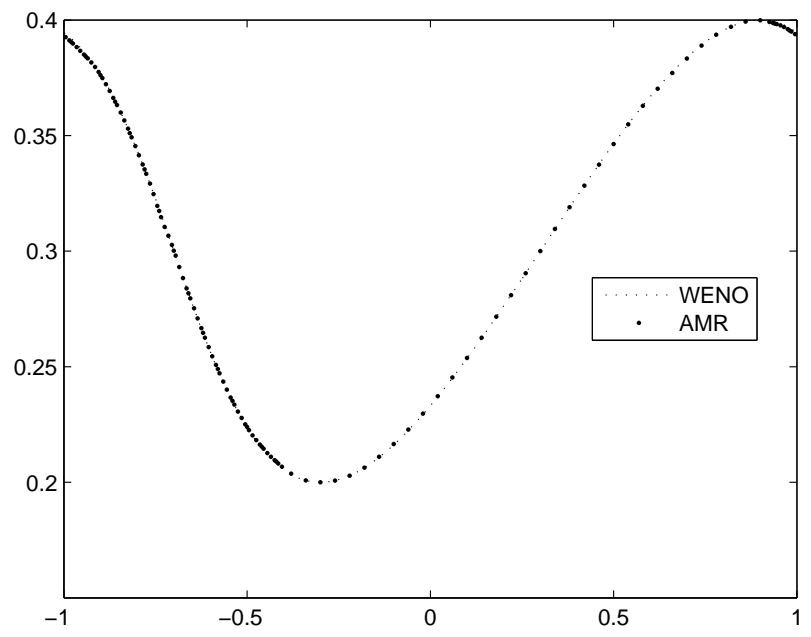

Figure 5. Solutions to the Burgers' equation at $t=1$ from the WENO scheme with $N=800$ points and the AMRimplemented WENO scheme with $N=50$ points.

Table 3 shows the computational costs for the WENO and AMR-implemented WENO schemes for the Burgers' equation for $t \leq 6$. Table 3 and Figure 6 show that the accuracy from the AMR-implemented WENO with $N=50$ is comparable to that from the WENO with $N=800$, while the computational cost is reduced by about $90 \%$ when AMR is applied, similarly to the linear advection in Section 3.1.

TABLE 3. Computational costs for the WENO and AMRimplemented WENO schemes for the Burgers' equation for $t \leq 6$

\begin{tabular}{c|cc}
$t$ & WENO $(N=800)$ & AMR $(N=50)$ \\
\hline 1 & $968 \mathrm{~ms}$ & $109 \mathrm{~ms}$ \\
2 & $1 \sec 922 \mathrm{~ms}$ & $140 \mathrm{~ms}$ \\
3 & $2 \sec 875 \mathrm{~ms}$ & $265 \mathrm{~ms}$ \\
4 & $3 \sec 844 \mathrm{~ms}$ & $390 \mathrm{~ms}$ \\
5 & $4 \sec 797 \mathrm{~ms}$ & $516 \mathrm{~ms}$ \\
6 & $5 \sec 687 \mathrm{~ms}$ & $578 \mathrm{~ms}$ \\
\hline
\end{tabular}




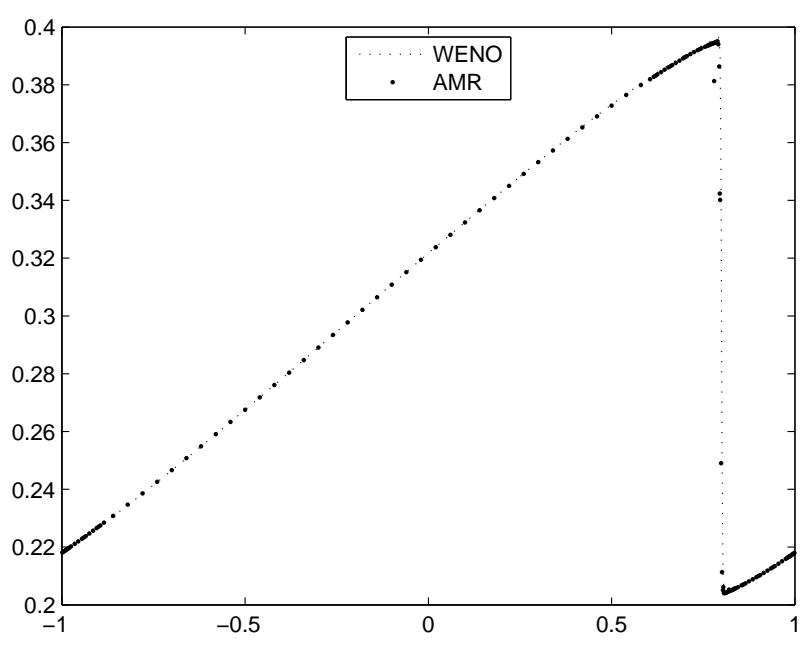

Figure 6. Solutions to the Burgers' equation at $t=6$ from the WENO scheme with $N=800$ points and the AMRimplemented WENO scheme with $N=50$ points.

\subsection{Euler equation}

In this section we solve the one-dimensional Euler equation [16]

$$
\left(\begin{array}{c}
\rho \\
\rho v \\
E
\end{array}\right)_{t}+\left(\begin{array}{c}
\rho v \\
\rho v^{2}+p \\
v(E+p)
\end{array}\right)_{x}=0
$$

for $x \in[0,1]$, where $\rho$ is the density, $v$ is the velocity, $E$ is the total energy, and $p$ is the pressure. The total energy $E$ is decomposed as

$$
E=\frac{1}{2} \rho v^{2}+\rho e
$$

for the specific internal energy $e$. See [16] for details. Following initial conditions are used:

$$
(\rho, v, p)= \begin{cases}(0.125,0.0,0.1) & \text { if } x>0.3 \\ (1.0,0.75,1.0) & \text { if } x<0.3\end{cases}
$$

The CFL number is fixed to 0.9 and $\gamma=1.4$.

When the problem is solved for $t \leq 0.2$, the numerical solutions for $e, p, \rho$ and $v$ are obtained as in Figure 7 and Figure 8. As in the previous examples, the AMR-implemented WENO with $N=50$ points gives quite accurate results. We can infer from these numerical experiments that the derived AMRimplemented WENO scheme is also efficient for solving this nonlinear system 
of equations whose solutions contain rarefactions and contact discontinuities as well as shocks.
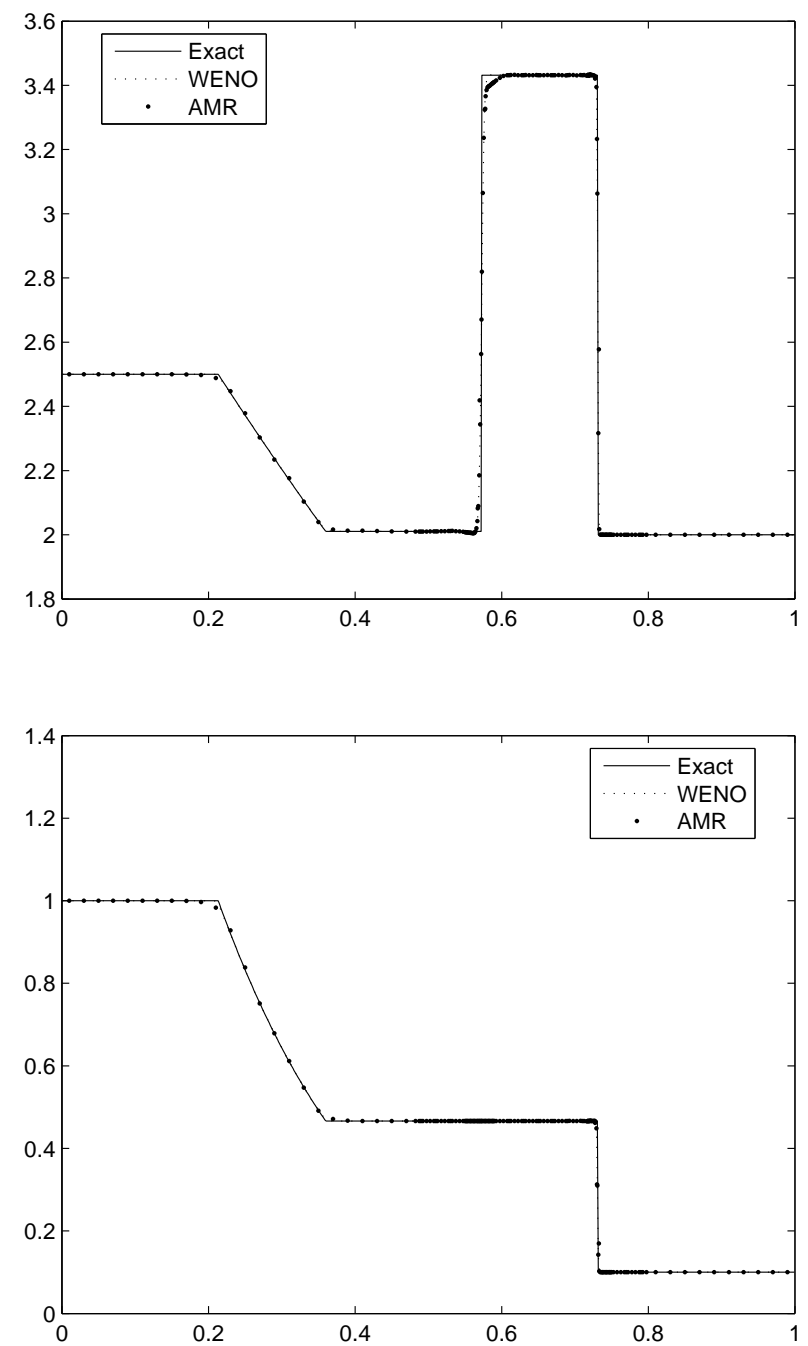

Figure 7. Solutions to the Euler equation at $t=0.2$ from the WENO scheme with $N=800$ points and the AMRimplemented WENO scheme with $N=50$ points. (Top) $e$ (Bottom) $p$ 

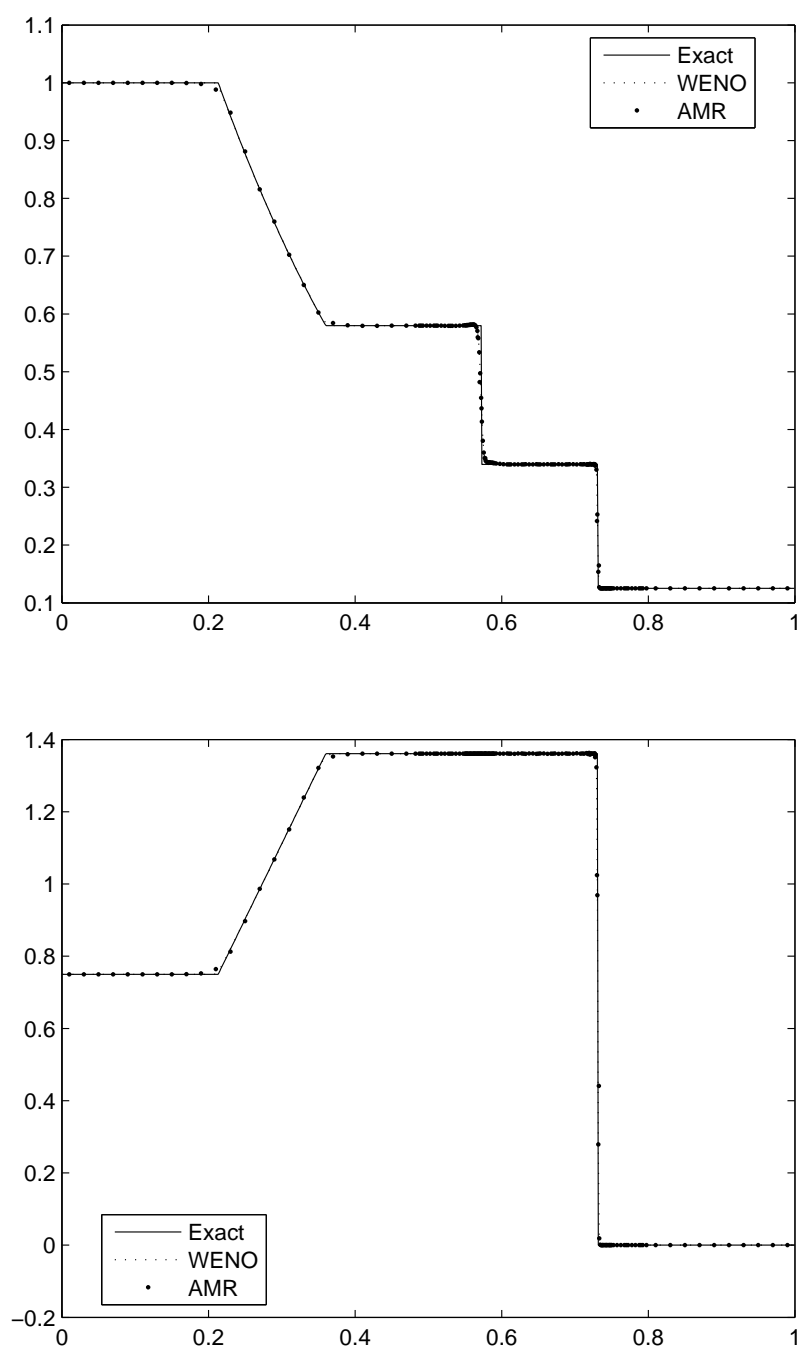

Figure 8. Solutions to the Euler equation at $t=0.2$ from the WENO scheme with $N=800$ points and the AMRimplemented WENO scheme with $N=50$ points. (Top) $\rho$ (Bottom) $v$

Table 4 shows the computational costs for the WENO and AMR-implemented WENO schemes for the Euler equation for $t \leq 0.2$. It shows that about $90 \%$ of the computational cost is reduced due to the implementation of AMR. 
TABLE 4. Computational costs for the WENO and AMRimplemented WENO schemes for the Euler equation for $t \leq 0.2$

\begin{tabular}{c|cc}
$t$ & WENO $(N=800)$ & $\operatorname{AMR}(N=50)$ \\
\hline 0.2 & $12 \sec 594 \mathrm{~ms}$ & $1 \sec 500 \mathrm{~ms}$ \\
\hline
\end{tabular}

\section{Conclusions}

AMR has been successfully implemented to the WENO scheme to solve linear or nonlinear equations containing discontinuities. When AMR is applied to the WENO scheme, the computational cost can be reduced while the accuracy is maintained. In our experiments, the cost is reduced by $90 \%$. In the future research, the AMR will be implemented over a hybrid WENO scheme. For example, the AMR with the central difference may be applied over smooth region while the AMR with the WENO near discontinuities.

\section{References}

[1] G. Jiang and C. W. Shu, Efficient implementation of weighted ENO schemes, J. Comput. Phys. 126 (1996), no. 1, 202-228.

[2] D. Kim and J. H. Kwon, A high-order accurate hybrid scheme using a central flux scheme and a WENO scheme for compressible flowfield analysis, J. Comput. Phys. 210 (2005), no. 2, 554-583.

[3] C. W. Shu, Essentially non-oscillatory and weighted essentially non-oscillatory schemes for hyperbolic conservation laws, NASA/CR-97-206253, ICASE Report No. 97-65, 1997.

[4] D. Balsara and C. W. Shu, Monotonicity preserving weighted essentially non-oscillatory schemes with increasingly high order of accuracy, J. Comput. Phys. 160 (2000), no. 2, $405-452$.

[5] J. Shi, C. Hu, and C. W. Shu, A technique of treating negative weights in weno schemes, J. Comput. Phys. 175, (2002), 108-127.

[6] R. Wang, H. Feng, and R. J. Spiteri, Observations on the fifth-order WENO method with non-uniform meshes, Appl. Math. Comput. 196 (2008), no. 1, 433-447.

[7] M. Berger and J. Oliger, Adaptive mesh refinement for hyperbolic partial differential equations, J. Comput. Phys. 53 (1984), no. 3, 484-512.

[8] M. Berger and P. Colella, Local adaptive mesh refinement for shock hydrodynamics, J. Comput. Phys. 82 (1989), 67-84.

[9] J. Glimm, H. Kim, D. Sharp, and T. Wallstrom, A stochastic analysis of the scale up problem for flow in porous media, Comput. Appl. Math. 17 (1998), no. 1, 67-79.

[10] C. W. Shu and S. Osher, Efficient implementation of essentially nonoscillatory shockcapturing schemes. II., J. Comput. Phys. 83 (1989), no. 1, 32-78.

[11] R. Deiterding, Parallel adaptive simulation of multi-dimensional detonation structures, Ph. D. thesis, Brandenburgische Technische Universität Cottbus, 2003.

[12] M. Berger and I. Rigoutsos, An algorithm for point clustering and grid generation, IEEE Trans. on System. 21 (1991), no. 5, 1278-1286.

[13] S. Li and J. M. Hyman, Adaptive mesh refinement for finite difference weno schemes, Technical Report LA-UR-03-8927, Los Alamos National Lab, 2003.

[14] J. M. Hyman and S. Li, Interactive and dynamic control of adaptive mesh refinement with nested hierarchical grids, Technical Report LA-UR-98-5462, Los Alamos National Lab, 1998. 
[15] M. J. Berger and R. J. Leveque, Adaptive mesh refinement using wave-propagation algorithms for hyperbolic systems, SIAM J. Numer. Anal. 35 (1998), no. 6, 2298-2316.

[16] R. J. LeVeque, Numerical Methods for Conservation Laws, Birkhäuser, 1992.

\section{DAEKI YOON}

Department of Mathematics

Korea UNIVERSITY

SEOUl 136-701, KoreA

E-mail address: dyoon@korea.ac.kr

HONGJOONG KIM

Department of Mathematics

Korea UNIVERSITY

SEOUl 136-701, KoreA

E-mail address: hongjoong@korea.ac.kr

WoOnJae HWANG

Department of Information AND Mathematics

Korea UNIVERSITY

JOCHIWON 739-700, KorEA

E-mail address: woonjae@korea.ac.kr 